\title{
ON FIXED POINTS FOR SEMI-GROUPS OF LINEAR OPERATORS
}

\author{
BERTRAM YOOD
}

1. Introduction. The starting point of the present investigation is the following result of Dunford ${ }^{1}[3$, p. 8].

Theorem (DUNFORD). Let $G$ be a semi-group of bounded linear operators on a normed linear space $X$ where (a) $G$ is bounded, (b) $G$ is abelian, and (c) the elements of $G$ have a (common) nonzero fixed point. ${ }^{2}$ Then the semigroup $G^{*}=\left\{T^{*} \mid T \in G\right\}$ of adjoints of elements in $G$ has a nonzero fixed point (in $X^{*}$ ).

That such theorems, when applied to the space $X$ of bounded functions on a set $S$, have measure-theoretic significance was noted in [3]. Let $H$ be a semi-group of 1-1 transformations of $S$ into $S$. To each $\sigma \in H$ we correspond the transformation $T_{\sigma}$ of $X$ into itself where $T_{\sigma}(f)$ is the function $f(\sigma(s)), s \in S, f \in X$. These transformations form a semi-group $G$ each element of which has norm one. Furthermore $G$ has the unit function as a nonzero fixed point. If $G^{*}$ also possesses a nonzero fixed point $x_{0}^{*}$, then there is a bounded additive set function $\mu$ defined for all subsets of $S$ such that

$$
x_{0}^{*}(f)=\int_{S} f(t) d \mu, \quad f \in X,
$$

where the integral is taken in the sense of Hildebrandt [5]. Then using the characteristic function of the set $E \subset S$ in this formula, $\mu(E)=\mu(\sigma(E))$ for each $\sigma$ and each $E \subset S$. This equality persists if $\mu(E)$ is replaced by $\mu_{1}(E)$, the total variation of $\mu$ on $E$. Then $\mu_{1}$ has the properties that $\mu_{1}(E) \geqq 0$ for each subset $E$ of $S, \mu_{1}$ is finitely additive, $\mu_{1}(S) \neq 0$, and $\mu_{1}(E)=\mu_{1}(\sigma E)$ for each $\sigma \in H, E \subset S$. In particular the theorem of Dunford implies the existence of such a set function when $H$ is an abelian semi-group. von Neumann [7] has shown that such a set function must exist if $H$ is a solvable group or a

\footnotetext{
Presented to the Society, October 28, 1950; received by the editors January 9 , 1950.

1 This result is not stated in this form in [3] but follows readily from the arguments used there. The author is grateful to Professor Dunford for calling his attention to this result and suggesting the present investigation. Numbers in brackets refer to the bibliography at the end of the paper.

2 By this we mean that there exists an element $x_{0}$ in $X, x_{0} \neq 0$, such that $T\left(x_{0}\right)=x_{0}$ for each $T$ in $G$. We then say that $G$ possesses a nonzero fixed point.
} 
finite group of 1-1 transformations of $S$ onto $S$. This suggests that the theorem of Dunford may be valid if (b) is replaced by the statement that $G$ is a solvable group or a finite group. We find this to be the case. In fact, by Theorem 4, the result is true if instead of a finite group we use any group with the property that any finite subset of it lies in a finite subgroup.

While this motivates much of our work, we shall also be concerned with improving Dunford's result by replacing (a) and (c) with weaker conditions. (a) is replaced by a condition which covers some unbounded semi-groups and (c) is replaced by a necessary condition. In this connection we give the following example to show that, even in the case of a bounded semi-group $G$ with one generator, (c) is not necessary for the conclusion.

Let $X=(l)$ be the space of sequences $x=\left\{\xi_{i}\right\}$ for which $\sum_{i=1}^{\infty}\left|\xi_{i}\right|$ $<\infty$. Let $T$ be the transformation defined by the rule $T(x)=y$ where $x=\left\{\xi_{i}\right\}$ and $y=\left\{0, \xi_{1}, \xi_{2}, \cdots\right\}$. Let $G$ be the semi-group generated by $T$. It is readily seen that if $U(x)=x$ for all $U$ in $G$, then $x=\theta$, the zero element of $X$. On the other hand $T^{*}$ is a transformation defined on the space $(m)$ of bounded sequences to itself where if $x^{*}=\left\{\eta_{i}\right\}$ then $T^{*}\left(x^{*}\right)=\left\{\eta_{2}, \eta_{3}, \cdots\right\}$. Thus the element of $(m)$, all of whose coordinates are one, is a fixed point for $G^{*}$.

2. Notation. Let $G$ be a semi-group of bounded linear operators on a normed linear space $X$. We indicate by $G^{*}$ the semi-group consisting of all the adjoint operators $T^{*}, T \in G$. Also we let $G_{1}$ represent the collection of all averages of elements in $G$. It is clear that $G_{1}$ is a semi-group containing $G$, and that $\left(G_{1}\right)_{1}=G_{1}$. The following notation is convenient.

$A(G)=\{T(x)-x \mid T \in G, x \in X\}$.

$B(G)$ : the linear manifold generated by $A(G)$.

$D(G)=\left\{x \in X \mid \inf _{T \in G}\|T(x)\|>0\right\}$.

$Z(G)$ : the complement of $D(G)$.

We designate by $E(X)$ the collection of all bounded linear operators defined on $X$. We shall say that the semi-group $G$ satisfies the relaxed boundedness condition (a) if there is a number $K>0$ such that for each $T \in G_{1}$ there exists an operator $U \in E(X)$ such that $U T \in G_{1}$, $\|U\| \leqq K$, and $\|U T\| \leqq K$. $G$ will be said to satisfy the relaxed boundedness condition (b) if the $U$ of the preceding definition can always be selected from $G_{1}$. For brevity these conditions will be referred to as the r.b.c. (a) and r.b.c. (b) respectively. The number $K>0$ will hereafter be that appearing in the r.b.c. satisfied by $G$.

We give an example of a semi-group which is unbounded but satisfies the r.b.c. (b). Let $X$ be an infinite-dimensional Banach space 
and let $x_{1}^{*}, \cdots, x_{n}^{*}$ be $n$ linearly independent elements of $X^{*}$. Consider all complexes $\left\{y_{1}, \cdots, y_{n}\right\}$ of $n$ elements of $X$ where $x_{i}^{*}\left(y_{j}\right)=\delta_{i j}, i, j=1, \cdots, n$. Such complexes must exist. Let $G$ be the collection of all transformations of the form $T(x)=\sum_{i=1}^{n} x_{i}^{*}(x) y_{i}$. If $U, V \in G$, then $U V=U$ so that $G$ is a semi-group. Since the relation $U V=U$ also holds for $G_{1}, G$ satisfies the r.b.c. (b). But there exists an element $y_{0} \neq 0$ in $X$ such that $x_{i}^{*}\left(y_{0}\right)=0, i=1, \cdots, n$. If $T(x)=\sum_{i=1}^{n} x_{i}^{*}(x) y_{i}$ is in $G$, then so are the transformations $U_{m}(x)$ $=\sum_{i=1}^{n} x_{i}^{*}(x)\left[y_{i}+m y_{0}\right]$. Since $U_{m}\left(y_{1}\right)=y_{1}+m y_{0},\left\|U_{m}\right\| \rightarrow \infty$.

3. Some fixed point theorems. Our aim is to obtain sufficient conditions on the semi-group $G$ in $E(X)$ to insure that $G^{*}$ has a nonzero fixed point. To carry out the arguments we find it necessary to use a boundedness condition. Under the r.b.c. (b), (1) and (2) below are sufficient, (1) being necessary. It is then shown that (3) implies (2) and that abelian semi-groups and solvable groups satisfy (3) (under appropriate boundedness conditions). Some other results are also obtained.

LEMMA 1. Let $G$ be a semi-group in $E(X)$. A necessary and sufficient condition for $G^{*}$ to possess a nonzero fixed point is that $\overline{B(G)} \neq X$. It is necessary that

$$
D\left(G_{1}\right) \text { is not empty. }
$$

It is sufficient that (1) holds and also

$$
\overline{B(G)} \subset Z\left(G_{1}\right)
$$

Proof. The first statement is an easy consequence of the HahnBanach theorem $[2$, p. 55]. To see the necessity of (1), consider a nonzero fixed point $x_{0}^{*}$ of $G^{*}$. Since $x_{0}^{*}[T(x)]=x_{0}^{*}(x), x \in X, T \in G_{1}$, any element $x_{0}$ with the property that $x_{0}^{*}\left(x_{0}\right) \neq 0$ must be in $D\left(G_{1}\right)$.

That (1) and (2) are sufficient follows from the fact that they imply $\overline{B(G)} \neq X$.

In what follows we find sufficient conditions for (2) to hold. This task is lightened by the fact that in all our situations the r.b.c. (a) condition holds and, by the following lemma, there $Z\left(G_{1}\right)$ is closed and (2) may be replaced by the condition $B(G) \subset Z\left(G_{1}\right)$.

LemMa 2. Let $G$ be a semi-group in $E(X)$ satisfying the r.b.c. (a). Then $D\left(G_{1}\right)$ is open in $X$. If the r.b.c. (b) is fulfilled, $A(G) \subset Z\left(G_{1}\right)$.

Proof. In proving that $D\left(G_{1}\right)$ is open we may assume that neither $D\left(G_{1}\right)$ nor its complement $Z\left(G_{1}\right)$ is empty. Let $x_{0} \in D\left(G_{1}\right), y_{0} \in Z\left(G_{1}\right)$. Then inf $\left\|T\left(x_{0}\right)\right\|=\delta>0, T \in G_{1}$. We take $\epsilon<\delta$. There exists $T_{0} \in G_{1}$ 
such that $\left\|T_{0}\left(y_{0}\right)\right\|<\epsilon / K$, and by the r.b.c. (a) there exists a $U_{0}$ $\in E(X),\left\|U_{0}\right\| \leqq K,\left\|U_{0} T_{0}\right\| \leqq K, \quad U_{0} T_{0} \in G_{1}$. Thus $\left\|U_{0} T_{0}\left(y_{0}\right)\right\|<\epsilon$. However $\left\|U_{0} T_{0}\left(x_{0}\right)\right\| \geqq \delta$. Thus

$$
K\left\|x_{0}-y_{0}\right\| \geqq\left\|U_{0} T_{0}\left(x_{0}-y_{0}\right)\right\| \geqq \delta-\epsilon .
$$

Since $\epsilon>0$ is arbitrary, the distance from $x_{0}$ to $Z\left(G_{1}\right)$ is at least $\delta / K$. Hence $D\left(G_{1}\right)$ is open.

Suppose that the r.b.c. (b) holds. Let $T(x)-x \in A(G), T \in G$ and set $V=\left(T+\cdots+T^{r}\right) / r$ where $r$ is a positive integer. Now $V[T(x)$ $-x]=\left[T^{r+1}(x)-T(x)\right] / r$. By the r.b.c. (b) there exist operators $V_{i}$ in $G_{1}$ such that $\left\|V_{i}\right\| \leqq K, i=1,2,\left\|V_{1} T\right\| \leqq K$, and $\left\|V_{2} V_{1} T^{r+1}\right\| \leqq K$. Then

$$
\left\|V_{2} V_{1} V[T(x)-x]\right\| \leqq\left(K+K^{2}\right)\|x\| / r .
$$

This shows that $A(G) \subset Z\left(G_{1}\right)$.

THEOREM 1. Let $G$ be a semi-group in $E(X)$ satisfying the r.b.c. (a). Then (2) holds if

$$
A(G) \subset Z\left(G_{1}\right) \text { and } T[A(G)] \subset Z\left(G_{1}\right) \text { for each } T \in G_{1} \text {. }
$$

Proof. Take $\epsilon>0$. Let $y=\sum_{i=1}^{n} T_{i}\left(x_{i}\right)-x_{i}$ be any element in $B(G), T_{i} \in G, x_{i} \in X$. By hypothesis $y_{1}=T_{1}\left(x_{1}\right)-x_{1}$ is in $Z\left(G_{1}\right)$ and by the r.b.c. (a) there exists an element $V_{1} \in G_{1},\left\|V_{1}\right\| \leqq K$ such that $\left\|V_{1}\left(y_{1}\right)\right\|<\epsilon /\left(n K^{n}\right)$. Suppose that transformations $V_{i}$ in $G_{1}$ have been defined for $i=1, \cdots, m<n$ where $\left\|V_{i}\right\| \leqq K$ and for each such $i$, $\left\|V_{i} V_{i-1} \cdots V_{1}\left[T_{i}\left(x_{i}\right)-x_{i}\right]\right\|<\epsilon /\left(n K^{n}\right)$. Then by (3) $V_{m} \cdots V_{1}$ . $\left[T_{m+1}\left(x_{m+1}\right)-x_{m+1}\right]$ is in $Z\left(G_{1}\right)$ and by the r.b.c. (a) there exists $V_{m+1} \in G_{1}$ such that $\left\|V_{m+1} \cdots V_{1}\left[T_{m+1}\left(x_{m+1}\right)-x_{m+1}\right]\right\|<\epsilon /\left(n K^{n}\right)$. Thus $V_{1}, \cdots, V_{n}$ can be defined in turn with the above properties. Setting $V=V_{1} \cdots V_{n}$ we have $V \in G_{1}$ and

$$
\|V(y)\| \leqq \sum_{i=1}^{n}\left\|\left(V_{n} \cdots V_{i+1}\right)\left(V_{i} \cdots V_{1}\right)\left[T_{i}\left(x_{i}\right)-x_{i}\right]\right\|<\epsilon .
$$

This shows that $B(G) \subset Z\left(G_{1}\right)$. By Lemma 2, (2) is satisfied.

It may be remarked that the condition $A(G) \subset Z\left(G_{1}\right)$ is fulfilled if $T^{n} / n \rightarrow 0$ for each $T$ in $G$ or, by Lemma 2 , if $G$ satisfies the r.b.c. (b). Also we find it convenient to replace $A(G)$ by $A\left(G_{1}\right)$ in (3). As for the relation between $A(G)$ and $A\left(G_{1}\right)$, we have the following lemma.

LEMMA 3. If $G$ is a semi-group in $E(X)$, then $A\left(G_{1}\right) \subset B(G)$ and $B\left(G_{1}\right)$ $=B(G)$. 
Proof. Let $T(x)-x \in A\left(G_{1}\right), T=\left(T_{1}+\cdots+T_{n}\right) / n$ where each $T_{i} \in G$. Then $T(x)-x=\sum_{i=1}^{n}\left[T_{i}(x / n)-(x / n)\right]$. The remaining statement is clear.

THEOREM 2. Let $G$ be an abelian semi-group in $E(X)$ satisfying the r.b.c. (b). Then (3) of Theorem 1 is satisfied.

Proof. We show that $T\left[A\left(G_{1}\right)\right] \subset Z\left(G_{1}\right)$ for each $T \in G_{1}$ which with Lemma 2 clearly implies (3). Since $G$ is a commutative semigroup if $V(x)-x \in A\left(G_{1}\right)$ and $T \in G_{1}$, then $T[U(x)-x]=U T(x)-T(x)$ is in $A\left(G_{1}\right)$ and hence by Lemma 2 (for $G=G_{1}$ ) is in $Z\left(G_{1}\right)$.

We turn our attention next to the case of a solvable group $G$ in $E(X)$. The main steps in the argument will be given as lemmas.

LEMma 4. Let $G$ be a group in $E(X)$ and $H$ a normal subgroup of $G$. Then if $T \in G_{1}, T[B(H)] \subset B(H)$.

Proof. Let $V(x)-x, V \in H$, be one of the summands of an element in $B(H)$. It is sufficient to show that $T[V(x)-x] \in B(H)$. Now $T$ may be written in the form $\left(T_{1}+\cdots+T_{n}\right) / n, T_{i} \in G$, and

$$
T[V(x)-x]=\sum_{i=1}^{n} T_{i} V[x / n]-T_{i}(x / n) .
$$

Thus it is enough to prove that $T_{i}[V(y)-y] \in B(H), y \in X$. Actually we show that this is in $A(H)$.

Since $T_{t}^{-1}$ exists, there exists an element $z \in X$, such that $y=T_{t}^{-1}(z)$. Then $T_{i}[V(y)-y]=T_{i} V T_{i}^{-1}(z)-z$. But since $H$ is a normal subgroup of $G, T_{i} V T_{i}^{-1}$ is in $H$ and $T_{i}[V(y)-y]$ is in $A(H)$.

In particular this result holds if $H=G^{\prime}$, the derived subgroup of $G$.

Lемма 5. Let $G$ be a group in $E(X)$ and $G^{\prime}$ its derived group. If $S \in G_{1}, V \in G_{1}, x \in X$, then $S[V(x)-x]$ is equal to the (finite) sum of elements in $A\left(G^{\prime}\right)$ and an element in $A\left(G_{1}\right)$.

Proof. Let $I$ be the identity transformation in $E(X)$. We may write $S=\left(\sum_{i=1}^{n} S_{i}\right) / n, V=\left(\sum_{i=1}^{m} V_{i}\right) / m$ where $S_{i}, V_{i} \in G$. Then we have, setting $y=x /(m n)$,

$$
\begin{aligned}
S V(x)-S(x) & =\frac{1}{m n}\left[\sum_{i=1}^{n} S_{i} \sum_{j=1}^{m} V_{i}(x)-\sum_{i=1}^{n} S_{i} \sum_{j=1}^{m} I(x)\right] \\
& =\sum_{i=1}^{n} \sum_{j=1}^{m}\left[S_{i} V_{j}(y)-S_{i}(y)\right] .
\end{aligned}
$$

But for each pair $(i, j)$ we have 
$S_{i} V_{i}(y)-S_{i}(y)=\left[S_{i} V_{j} S_{i}^{-1} V_{j}^{-1}\left(V_{j} S_{i}\right) y-V_{j} S_{i}(y)\right]+\left[V_{j} S_{i}(y)-S_{i}(y)\right]$.

We note that the first summand on the right is in $A\left(G^{\prime}\right)$. Let $Q$ represent the sum on $i$ and $j$ of these terms in $A\left(G^{\prime}\right)$. Then

$$
\begin{aligned}
S V(x)-S(x) & =Q+\sum_{i=1}^{n} \sum_{j=1}^{m}\left[V_{j} S_{i}(y)-S_{i}(y)\right] \\
& =Q+\left[\frac{1}{m} \sum_{j=1}^{m} V_{j}\right]\left[\frac{1}{n} \sum_{i=1}^{n} S_{i}(x)\right]-\frac{1}{n} \sum_{i=1}^{n} S_{i}(x) .
\end{aligned}
$$

This completes the proof of the lemma.

LEMMA 6. Let $G$ be a group in $E(X)$ which satisfies the r.b.c. (b) and let $G^{\prime}$ be its derived group. If $B\left(G_{1}^{\prime}\right) \subset Z\left(G_{1}^{\prime}\right)$, then $T\left[A\left(G_{1}\right)\right] \subset Z\left(G_{1}\right)$ for each $T \in G_{1}$.

Proof. Let $y=V(x)-x \in A\left(G_{1}\right), T \in G_{1}, \epsilon>0$. By Lemma 5, $T(y)$ may be expressed as the sum of two elements $z+w$ where $z \in B\left(G^{\prime}\right)$ and $w \in A\left(G_{1}\right)$. By the use of Lemma 2 (for $G_{1}$ ), we see that there exists $W \in G_{1},\|W(w)\|<\epsilon /(2 K)$. By Lemma $4, W(z) \in B\left(G^{\prime}\right)$ and is thus in $Z\left(G_{1}^{\prime}\right)$, by hypothesis, and hence in $Z\left(G_{1}\right)$. By the r.b.c. (b), there exists $R \in G_{1},\|R\| \leqq K$ where $\|R W(z)\|<\epsilon / 2$. But then $\|R W T(y)\| \leqq\|R W(z)\|+\|R W(w)\|<\epsilon$ so that $T(y) \in Z\left(G_{1}\right)$.

We shall let $H^{(n)}$ represent the $n$th derived subgroup of a group $H$.

Theorem 3. Let $G$ be a solvable group in $E(X)$ where $G, G^{\prime}, G^{\prime \prime}, \ldots$ each satisfies the r.b.c. (b). Then $G$ satisfies (3).

Proof. Suppose $T\left[A\left(G_{1}^{(n)}\right)\right] \subset Z\left(G_{1}^{(n)}\right)$ for each $T \in G_{1}^{(n)}$. By Theorem 1, $B\left(G_{1}^{(n)}\right) \subset Z\left(G_{1}^{(n)}\right)$. Then by Lemma 6, $T\left[A\left(G_{1}^{n-1}\right)\right]$ $\subset Z\left(G_{1}^{(n-1)}\right)$ for all $T \in G_{1}^{(n-1)}$. As $G$ is solvable we obtain $T\left[A\left(G_{1}\right)\right]$ $\in Z\left(G_{1}\right)$ for all $T \in G_{1}$. Thus (3) is fulfilled by $G$.

CoROllary 1. Let $G$ be a solvable group in $E(X)$ where $G$ has the property that there exists a number $K>0$ such that

$$
\lim \sup \left\|T^{n}\right\| \leqq K, \quad T \in G_{1} .
$$

Then $G$ satisfies (3).

Proof. The condition of the corollary insures that $G^{(n)}$ satisfies the r.b.c. (b) for each $n$. Thus we may apply Theorem 3 .

TheOREM 4. Let $G$ be a group in $E(X)$ with the property that any finite subset of $G$ lies in a finite subgroup of $G$. Suppose that $Z\left(G_{1}\right)$ is closed. Then $G^{*}$ has a nonzero fixed point if (1) holds. 
Proof. We remark that by Lemma $2, Z\left(G_{1}\right)$ is closed if $G$ satisfies the r.b.c. (a). What we do show is that $B(G) \subset Z\left(G_{1}\right)$ and simply desire some hypothesis which will enable us to state the conclusion via Lemma 1.

Let $y=\sum_{i=1}^{n} T_{i}\left(x_{i}\right)-x_{i}$ be an arbitrary element in $B(G)$. Let $H=U_{1}, \cdots, U_{r}$ be a finite subgroup of $G$ containing $T_{1}, \cdots, T_{n}$. We set $V=\left(U_{1}+\cdots+U_{r}\right) / r$. As $H$ is a group, the elements $U_{j} T_{i}, j=1, \cdots, r$, range over $H$. Thus $V(y)=0$ and $B(G) \subset Z\left(G_{1}\right)$.

Corollary 2. Let $G$ be a finite group in $E(X)$. Then a necessary and sufficient condition for $G^{*}$ to possess a nonzero fixed point is that (1) holds.

Proof. By Lemma 1, we need only consider the sufficiency. $G$, being finite, is automatically bounded. Hence the hypotheses of Theorem 4 are satisfied by $G$.

Mazur [6, Theorem 1] has demonstrated the following. Let $U$ $\in E(X),\|U\|=1$. Let $p$ be the number of linearly independent fixed points for $U, q$ that for $U^{*}$. Then $p \leqq q$. We generalize this result. In the situation of Mazur let $G$ be the semi-group generated by $U$. The linearly independent fixed points of $U$ are elements in $D\left(G_{1}\right)$ such that every nontrivial linear combination of them is in $D\left(G_{1}\right)$.

CoROllaRy 3. Let $G$ be any semi-group in $E(X)$ with the property that $\overline{B(G)} \subset Z\left(G_{1}\right)$. Let $x_{i}, i=1, \cdots, p$, be linearly independent elements in $D\left(G_{1}\right)$ such that every nontrivial linear combination of them is in $D\left(G_{1}\right)$. Then the number of linearly independent fixed points for $G^{*}$ is at least $p$.

Proof. We note that it has been shown above that the hypotheses on $G$ are satisfied by all the semi-groups (and groups) considered in Theorems 1-4. Also any set of fixed points for $G$ has the property demanded of the $x_{i}$ 's. Thus we have an extension of Mazur's result to all the situations treated above.

For the proof we note that the $x_{i}$ 's are linearly independent modulo $\overline{B(G)}$. Let $R$ denote the linear manifold generated by $x_{1}, \cdots$, $x_{p}$. Then $R$ and $\overline{B(G)}$ have only the zero element in common. The second portion of Mazur's proof of his theorem [6, pp. 14-15] may be applied verbatim to give the conclusion.

Alaoglu and Birkhoff $[1$, p. 299] have noted that if $X$ is uniformly convex and if $\|T\|=1$ for each $T$ in a semi-group $G$ of $E(X)$, then if (1) holds, $G$ has a nonzero fixed point. We obtain here the same conclusion, relaxing the norm condition and that on $X$ at the price of specializing $G$. 
Corollary 4. Let $G$ be a semi-group in $E(X)$ satisfying (1) and let $X$ be a reflexive space. If $G$ is abelian and satisfies the r.b.c. (b) or if $G$ is a solvable group satisfying (4), then $G$ has a nonzero fixed point.

Proof. By Lemma 1, Theorems 1 and 2, and Corollary 1, in either case $G^{*}$ has a nonzero fixed point $x_{0}^{*}$. Note that $x_{0}^{*}$ satisfies (1) for $G^{*}$. In the abelian case, as the adjoint operation preserves norm, $G^{*}$ also satisfies a relaxed boundedness condition. In the case of the solvable group $G$, by the same argument $G^{*}$ satisfies (4). Also as the adjoint operation preserves the inverse operation and is an antiisomorphism, $G^{*}$ is solvable since $G$ is. As above, $G^{* *}=\left\{T^{* *} \mid T \in G\right\}$ possess a nonzero fixed point. Since $X$ is reflexive and $T^{* *}=T$, this is also true for $G$.

4. On the problem of measure. We return to the discussion of the application of our results to the problem of the existence of measures. We adopt the following notation of von Neumann $[7$, p. 78]. Let $S$ be a set, $P$ a subset of $S$, and $H$ a group of 1-1 transformations of $S$ into $S$. We say that an $[S, P, H]$ measure $\mu$ exists if $\mu$ is defined for all subsets of $S, \mu$ is finitely additive, $\mu(\sigma E)=\mu(E)$ for all subsets $E \subset S, \sigma \in H$ and $\mu(P)=1 .^{3}$

We define $X$ and $G$ as in $\S 1$ and note that $G$ is bounded and automatically satisfies (1).

Theorem 5. Let $G$ have the property that $B(G) \subset Z\left(G_{1}\right)$. Then an $[S, P, H]$ measure exists if and only if there exists a number $\epsilon>0$ such that for each finite collection of elements $\sigma_{1}, \cdots, \sigma_{n}$ of $H$ there exists a point $s \in S$ where

$$
\frac{\text { the number of } i \text { 's for which } s \in \sigma_{i} P}{n} \geqq \epsilon \text {. }
$$

Proof. This situation covers the cases of a solvable group or a group with the property of Theorem 4. Suppose such a measure $\mu$ exists. Let $\phi^{(E)}(s)$ represent the characteristic function of the set $E$. Then the function $f(s)=\left[\phi^{\left(\sigma_{1} P\right)}(s)+\cdots+\phi^{\left(\sigma_{n} P\right)}(s)\right] / n$ has the property that $\int_{S} f(s) d \mu=1$. Since $\mu(P)=1$ and $f(s) \geqq 0$ for all $s \in S$, it is impossible that $f(s)<1 / \mu(S)$ for each $s \in S$.

Conversely suppose that there is such an $\epsilon>0$. For each $T \in G_{1}$, there exists $\sigma_{1}, \cdots, \sigma_{n} \in H$ such that $T\left[\phi^{(P)}\right]=\left[\phi^{\left(\sigma_{1}^{-1} P\right)}+\cdots\right.$ $\left.+\phi^{\left(\sigma_{n}^{-1} P\right)}\right] / n$. Then by hypothesis $\left\|T\left[\phi^{(P)}\right]\right\| \geqq \epsilon$ so that $\phi^{(P)} \in D\left(G_{1}\right)$.

${ }^{3}$ We consider only measures $\mu$ for which $\mu(S)<\infty$. 
Since $B(G) \subset Z\left(G_{1}\right)$, by Lemmas 1 and 2 and the arguments of $\S 1$, the required measure function exists.

5. On semi-groups in Banach algebras. We assume in this section that $X$ is a commutative Banach algebra (normed ring) with a unit $e$, $\|e\|=1$. Let $M$ be any maximal ideal of $X$ and let $x(M)$ be the homomorphism of $X$ onto the complex number system determined by $M$ (in the notation of Gelfand [4]). Then the set $S(M)=\{x \in X \mid x(M)$ $=1$ f forms a semi-group in $X$ which is also a hyperplane. We characterize here all sub-semi-groups of these semi-groups which satisfy the r.b.c. (b) suitably interpreted.

TheOREM 6. Let $G$ be a semi-group of elements in the Banach algebra $X$ and let $G_{1}$ be the collection of averages of elements in $G$. Suppose that there is a number $K>0$ such that for each $a \in G_{1}$ there corresponds an element $b \in G_{1},\|b\| \leqq K$, and $\|a b\| \leqq K$. Then a necessary and sufficient condition that there exists a maximal ideal $M$ of $X$ such that $G \subset S(M)$ is that inf $\|a\|>0, a \in G_{1}$.

Proof. The correspondence $a \leftrightarrow T_{a}$ where $T_{a}$ is the operation of left multiplication by the element $a$ takes $G$ into a semi-group $G^{\prime}$ of bounded linear operators on $X$. Furthermore, by [4], $\|a\|=\left\|T_{a}\right\|$. Thus under the conditions of the theorem, $G^{\prime}$ satisfies the r.b.c. (b). Also since $T_{a}(e)=a$ for each $a \in G_{1}, G^{\prime}$ satisfies (1). Hence by Theorem $2, B\left(G^{\prime}\right)$ is not dense in $X$. However it is readily verified that $B\left(G^{\prime}\right)$ is an ideal in $X$. Hence by [4] there exists a maximal ideal $M$ of $X$ which contains $B\left(G^{\prime}\right)$. For each $a \in G, b \in X$, we have $a b(M)=b(M)$. Since $(a b)(M)=a(M) b(M)$ (see [4]) and since $b$ is arbitrary in $X$, $a(M)=1$. The necessity follows from Lemma 1 .

\section{BibliogRAPHY}

1. L. Alaoglu and G. Birkhoff, General ergodic theorems, Ann. of Math. vol. 41 (1940) pp. 293-309.

2. S. Banach, Théorie des opérations lineaires, Warsaw, 1932.

3. N. Dunford, Direct decompositions of Banach spaces, Boletin de la Sociedad Matematica Mexicana vol. 3 (1946) pp. 1-12.

4. I. Gelfand, Normierte Ringe, Rec. Math. (Mat. Sbornik) N.S. vol. 9 (1941) pp. 3-24.

5. T. H. Hildebrandt, On bounded linear functional operations, Trans. Amer. Math. Soc. vol. 36 (1934) pp. 868-875.

6. S. Mazur, Über die Nullstellen linearer Operationen, Studia Mathematica vol. 2 (1930) pp. 11-20.

7. J. von Neumann, Zur allgemeinen Theorie des Masses, Fund. Math. vol. 13 (1929) pp. 73-116.

CoRnell UNIVERSity 